\title{
Effects of Two Types of Linearly Increased Isometric Exercise on Instantaneous Baroreflex and Respiratory Sinus Arrhythmia Sensitivities Computed by Alpha Index
}

\author{
Alejandra Guillén-Mandujano, Salvador Carrasco-Sosa \\ División Ciencias Biológicas y de la Salud, Universidad Autónoma Metropolitana-I, CDMX, México
}

\begin{abstract}
To assess the linearity of the relation between muscular force (\%MF) and baroreflex sensitivity (BRS), respiratory sinus arrhythmia sensitivity (RSAS) and their respective coherences (cBRS and cRSAS), 35 healthy subjects performed static handgrip $(H G)$ or leg extension (LE) linearly increased until fatigue (LIF). From the time-frequency spectra of $R-R$ intervals $(R R)$, respiration $($ Res), systolic $(S P)$ and diastolic pressures $(D P)$ series, their instantaneous low-frequency $\left(L F_{R R}, L F_{S P}, L F_{D P}\right)$ and high-frequency $\left(H F_{R R}, H F_{R e s}\right)$ powers were estimated to compute, by alpha index, BRS and RSAS, as well as their time-frequency coherences. Relations of \%MF with BRS, $c B R S$, RSAS and cRSAS were inverse, and those with heart rate (HR), DP and $H F_{\text {Res }}$, were direct, all but RSAS with greater slopes and correlations in LE than $H G$. RSAS can be computed analogously to BRS by alpha index, and their coherences can be used as complementary measures of the degree of their inputoutput coupling. Performing HG and LE in a LIF format provokes moderately to strongly correlated effects, greater for $L E$ than $H G$, consisting in progressive vagal withdrawal with loss of respiratory-cardiac modulation and release of sympathetic activity that cause the parallel increases of DP and $H R$ with increasing regularity, greater for $L E$ than $H G$.
\end{abstract}

\section{Introduction}

Previous studies of the association between isometric exercise (IE) intensity and its autonomic, cardiovascular and respiratory (ACR) effects have reported, in healthy young subjects, the reduction of baroreflex sensitivity (BRS) computed by the sequence method, provoked by the execution of a single load of static handgrip (HG) at $30 \%$ of maximal voluntary contraction (MVC) sustained for $3 \mathrm{~min}$ [1]. Reduced respiratory sinus arrhythmia (RSA), estimated by spectral measures of HRV, has been documented in healthy subjects performing $\mathrm{HG}$ at $40 \%$ MVC for 4 min [2]. In addition, the ACR effects of $\mathrm{HG}$ and leg extension (LE) have rarely been compared [3]. Thus, the relations of BRS and RSA with different intensities and types of IE remain unclear. To provide insight into this issue, we propose a methodological approach that involves: the performance of linearly increased until fatigue IE (LIFIE) to test the linearity of the BRS and RSA sensitivity (RSAS) responses across their entire operational range, computing RSAS by alpha index, analogously as it has been done with BRS, and comparing the responses to two types of LIFIE. Therefore, using the time-frequency analysis of cardiovascular variability that provides the instantaneous time course of its spectral measures, this study aimed at assessing BRS, RSAS and their respective coherences, during the performance of two types of LIFIE, HG and LE, and their correlation with the muscular force (MF) produced.

\section{Methods}

\subsection{Subjects}

Thirty five young, healthy, nonsmoking and sedentary subjects, 25 men and 10 women, participated. Their age, height and weight were $22.4 \pm 2.7$ years, $167 \pm 8 \mathrm{~cm}$ and $66.2 \pm 11.0 \mathrm{~kg}$ respectively. Their written informed consent was requested to participate. This study was approved by the ethics committee of our university.

\subsection{Protocol}

In a first visit to the laboratory, the health status of the subjects was evaluated and they were trained to correctly execute LIFIE. In a second visit, subjects performed static $\mathrm{HG}$ and LE in sitting position, resting for at least $30 \mathrm{~min}$. between trials. Each session consisted on three successive stages: 1 min. of control; subject performing incremental $\mathrm{HG}$ or $\mathrm{LE}$ at equal rate of $0.21 \mathrm{~kg} \cdot \mathrm{s}^{-1}$ until exhaustion, where the maximal MF was attained; and recovery for 2 min. Subjects linearly increased their MF following a target linear pattern visually presented. 


\subsection{Signal recording and acquisition}

ECG was detected at the thoracic bipolar lead CM5 using a bioelectric amplifier (Biopac Systems). Arterial pressure (AP) was recorded by Finapres (Ohmeda). Respiratory movements were recorded with a pneumograph (Nihon Kohden). MF was measured with two handgrip dynamometers (Stoelting), one mechanically adapted to a chair to measure MF in LE. All signals were digitized at a sampling rate of $1 \mathrm{kHz}$ via an acquisition and display system (Biopac Systems).

\subsection{Data processing}

Maxima and minima of ECG, AP and respiratory movements recordings were detected to construct the RR, systolic pressure (SP), diastolic pressure (DP) and respiration (Res) time series, which were cubic-spline interpolated, resampled at $4 \mathrm{~Hz}$ and detrended. Timefrequency spectra of these series were estimated with the smoothed pseudo-Wigner-Ville distribution and integrated in the standard frequency bands of HRV analysis to compute the instantaneous values of their low frequency $\left(\mathrm{LF}_{\mathrm{RR}}, \mathrm{LF}_{\mathrm{SP}}\right.$ and $\left.\mathrm{LF}_{\mathrm{DP}}\right)$ and high frequency $\left(\mathrm{HF}_{\mathrm{RR}}, \mathrm{HF}_{\mathrm{Res}}\right)$ powers. The gain of RSA, assumed as a system whose input is Res and its output is HRV, has been computed by the transfer function method [4], and now we propose to compute its time course by the alpha index, that is, as the square root of the $\mathrm{HF}_{\mathrm{RR}} / \mathrm{HF}_{\mathrm{Res}}$ ratio, as it is done with the $\mathrm{LF}_{\mathrm{RR}} / \mathrm{LF}_{\mathrm{SP}}$ ratio for BRS [5]. Their corresponding time-frequency coherences (cRSAS and cBRS) were computed from their respective spectral powers as the cross-spectrum divided by the square root of the product of the auto-spectra. Indexes dynamics of each subject were expressed as changes from mean baseline and ensemble-averaged for visualization. MF was normalized for the individual maximal force and expressed as percentage (\%MF). In case the continuous relation showed an inflection point, this threshold effect was detected by the V-slope method.

\subsection{Statistical analysis}

Data are expressed as mean \pm SD. Indexes dynamics during the two tests were used to compute linear regressions and correlations for each subject, before and after threshold when appropriate. Student's paired $t$-test was employed to compare the slopes (as index of the magnitude of the effect) and correlations between HG and LE. Statistical significance was accepted at $\mathrm{p}<0.05$.

\section{Results}

During HG and LE, the continuously increased MF: presented slopes around $0.21 \mathrm{~kg} / \mathrm{s}$, achieved similar maximal values $(\mathrm{HG}=26 \pm 4, \mathrm{LE}=27 \pm 3 \mathrm{~kg}$ ), and presented linear correlations with time close to 1 .

As MF increased linearly until fatigue (Fig. 1D), the time course of the series of: level and low frequency oscillations of DP progressively increased (Fig. 1A), level and high frequency oscillations of RR gradually decreased (Fig.1B), and amplitude and frequency of Res increased in the last third of LIFIE (Fig. 1C).

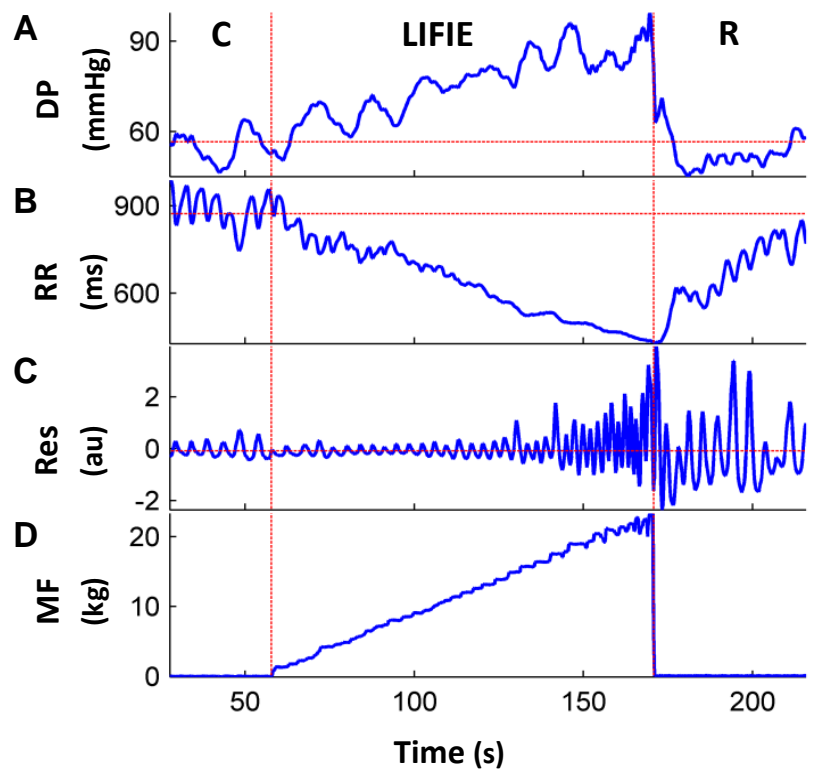

Fig. 1. Representative example of the progressive changes in the time series during LIFIE for LE. A) DP, B) RR, C) Res, D) MF. C = control, $\mathrm{R}=$ recovery.

The slopes of the linear regressions and correlations of the relations between \% MF and the responses of ACR measures to the two types of LIFIE, were:

- Direct for heart rate (HR), with greater slope and correlation in LE $(p<0.04)$ than HG (Fig. 2A).

- Direct for DP, with inflection points located between 67 and $69 \% \mathrm{MF}$ (Fig. 2B). The threshold effect was clearer in HG than LE. Correlations and slopes were greater for LE than HG (Table 1).

- Inverse for BRS, with greater slope and correlation in LE than HG ( $\mathrm{p}<0.04$, Fig. $2 \mathrm{C})$.

- Inverse for cBRS, presenting inflection points situated between 89 and 92\%MF (Fig. 2D), with similar slopes and greater correlations in LE (Table 1). Mean value of the baseline of the two types of LIFIE was $0.89 \pm 0.06$.

- Inverse for RSAS (Fig. 2E). Correlations and slopes were similar in $\mathrm{HG}$ and LE.

- Inverse for cRSAS (Fig. 2F), with inflection points located around $90 \% \mathrm{MF}$ and similar correlations and slopes (Table 1). Mean value of the baseline of the two types of LIFIE was $0.85 \pm 0.09$.

- Direct for $\mathrm{HF}_{\text {Res }}$, with inflection points situated between 67 and 75\% MF (Fig. 2G) and greater correlations and 
slopes in LE after \%MF threshold (Table 1).

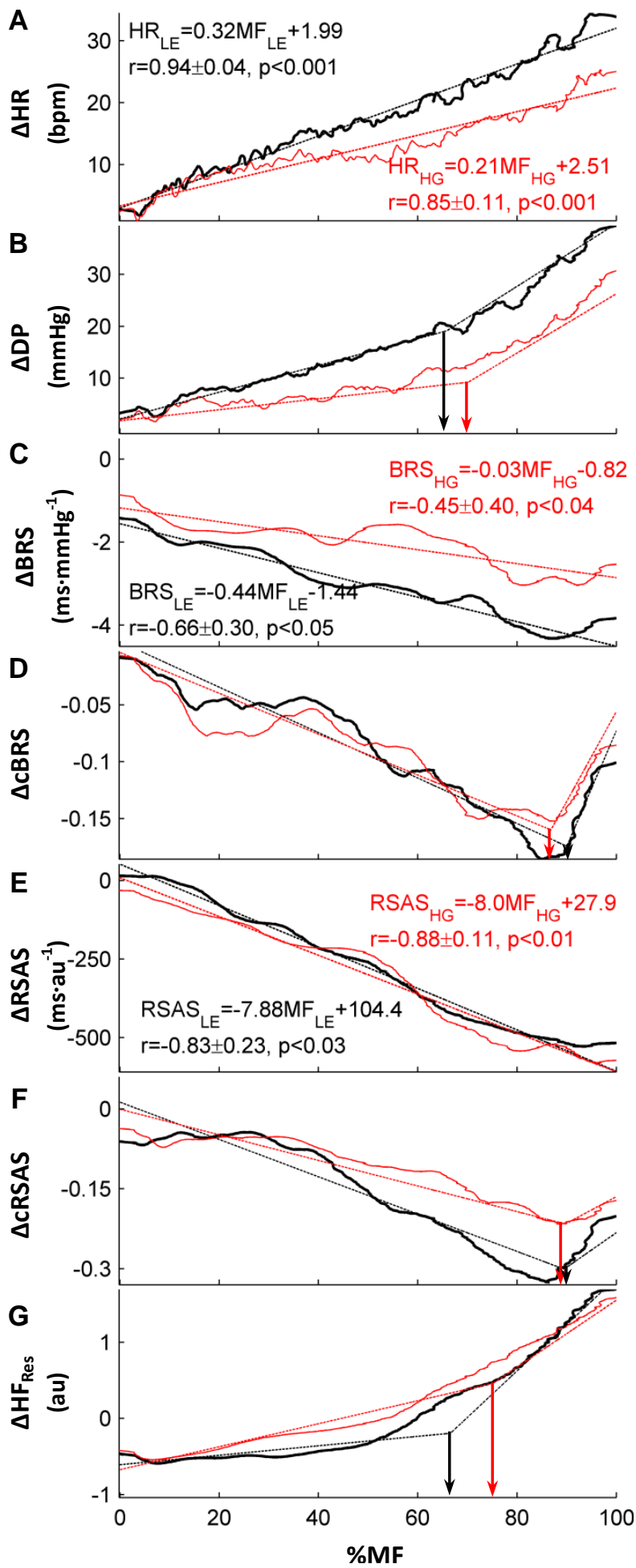

Fig. 2. Ensemble averages (solid lines), mean regressions (broken lines) and correlations of the continuous relations of \%MF with: A)HR, B)DP, C)BRS, D)cBRS, E) RSAS, F) cRSAS and G) $\mathrm{HF}_{\text {Res }}$ during LE (thick black lines) and HG (thin red lines). $\downarrow$ mark the inflection points in \%MF.
Table 1. Correlations (r) and regression slopes $(\mathrm{m})$ of the relations of \%MF with DP, cBRS, cRSAS and $\mathrm{HF}_{\mathrm{Res}}$ for both IE types before (BT) and after threshold (AT) \% MF. Mean $\pm \mathrm{SD}, \mathrm{N}=35$.

\begin{tabular}{cccccc}
\hline \multicolumn{5}{c}{} & \multicolumn{2}{c}{$\mathrm{BT}$} \\
$\mathrm{DP}$ & $\mathrm{r}$ & $0.76 \pm 0.12$ & $0.47 \pm 0.22^{*}$ & $0.80 \pm 0.09$ & $0.76 \pm 0.14$ \\
$\mathrm{mmHg}$ & $\mathrm{m}$ & $0.26 \pm 0.09$ & $0.12 \pm 0.06^{*}$ & $0.60 \pm 0.25$ & $0.53 \pm 0.19$ \\
$\mathrm{cBRS}$ & $\mathrm{r}$ & $-0.52 \pm 0.23$ & $-0.38 \pm 0.44^{*}$ & $0.68 \pm 0.35$ & $0.70 \pm 0.33$ \\
& $\mathrm{~m}$ & $-1.5 \pm 1.4 \dagger$ & $-1.4 \pm 1.8 \dagger$ & $7.4 \pm 11.2 \dagger$ & $6.9 \pm 8.3 \dagger$ \\
$\mathrm{cRSAS}$ & $\mathrm{r}$ & $-0.64 \pm 0.31$ & $-0.56 \pm 0.31$ & $0.39 \pm 0.68$ & $0.56 \pm 0.44$ \\
& $\mathrm{~m}$ & $-2.9 \pm 2.8 \dagger$ & $-2.1 \pm 2.2 \dagger$ & $7.8 \pm 8.1 \dagger$ & $5.2 \pm 6.1 \dagger$ \\
$\mathrm{HF}_{\mathrm{Res}}$ & $\mathrm{r}$ & $0.16 \pm 0.74$ & $0.77 \pm 0.25^{*}$ & $0.92 \pm 0.06$ & $0.87 \pm 0.10^{*}$ \\
$\mathrm{au}^{2}$ & $\mathrm{~m}$ & $4.8 \pm 20.1 \dagger$ & $13.0 \pm 9.9 * \dagger$ & $55.4 \pm 22.9 \dagger$ & $46.2 \pm 24.2 * \dagger$ \\
\hline$* \mathrm{p}<0.03$ between LE and HG. $\dagger \times 10^{-3}$ &
\end{tabular}

Mean regressions and correlations of BRS-cBRS and RSAS-cRSAS relations are shown in Fig. 3. BRS-cBRS correlation was greater in LE than $\mathrm{HG}(\mathrm{p}<0.05)$.
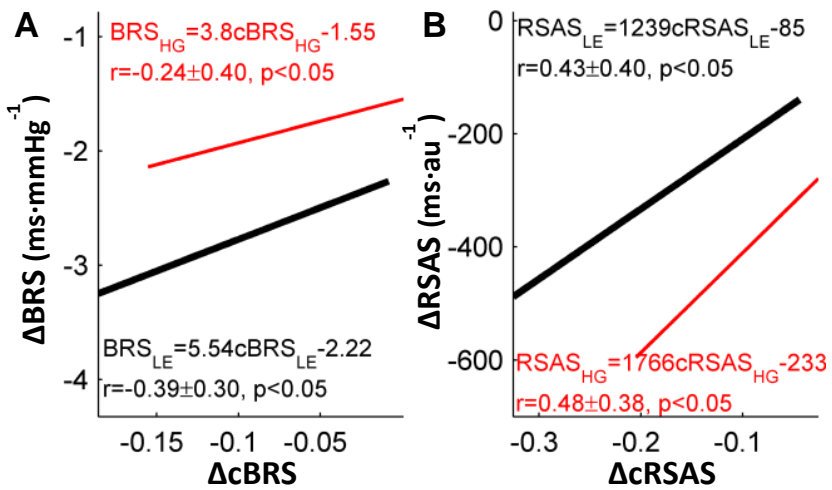

Fig. 3. Mean regressions and correlations between: A) BRS and cBRS, and B) RSAS and cRSAS during LE (black thick line) and HG (red thin line).

\section{Discussion}

Our methodological approach that comprises the performance of two types of LIFIE to test the linearity of the responses of BRS, RSAS, cBRS and cRSAS across their entire operational range, and computing RSAS and BRS by alpha index, widens the knowledge of these two relevant ACR control mechanisms, baroreflex (BR) and RSA, as supported by our main findings: In response to the linear \%MF increment, BRS and RSAS progressively decrease and HR shows gradual increase, with greater slopes and correlations in LE than HG, except for RSAS where they are similar; DP and $\mathrm{HF}_{\text {Res }}$ increase gradually, cBRS and cRSAS decrease, but these four last measures present a threshold effect in the last third of LIFIE, which is clearly apparent and is easily detected because the relations are continuous. The significant positive correlations between sensitivities and their coherences (Fig. 3) suggest their inherent functional interaction that 
supports the use of coherence as an index of their degree of input-output coupling.

There is consensus that IE provokes depression in BRS and RSA, computed from spectral measures of cardiovascular variability [1-3,6,7]. These effects have been repeatedly documented using a single load of HG at 30 to $40 \%$ MVC held for 2-4 min and computing BRS by sequence method $[1,2,6]$. Less frequently, LE execution [3] and BRS estimation by transfer function [7] have been employed. The comparison of the effects of LE and HG at $30 \%$ MVC has shown that LE elicits greater BRS reduction, attributed to the difference in the muscular mass involved [3]. RSAS better characterizes RSA, usually estimated by $\mathrm{HF}_{\mathrm{RR}}$, by quantitatively assessing the respiratory influence that determines it. cBRS has been employed as a validity criterion for computing BRS by frequency domain methods; when cBRS is less than 0.5 , it suggests the uncoupling of BR modulation of sinus node [8], as has been documented to occur when performing $\mathrm{HG}$ at $35 \% \mathrm{MVC}$ [7]. Our findings support that: the time course of the degree of BRS and RSAS input-output coupling is highly fluctuating, and there is a functional interaction between sensitivities and coherences (Fig. 3C-D) that turn them into complementary measures of autonomic control mechanisms. The reported relations between a single level of BRS and $\mathrm{HF}_{\mathrm{RR}}$ reductions provoked by one load of IE contrast with our continuous, moderately to strongly correlated relations of BRS and RSAS with \%MF in their widest possible range of function.

A functional explanation of our findings is that the visually-guided central command (CC) increases $\mathrm{MF}$ linearly until fatigue (Fig. 1D) and in parallel elicits: 1) gradual vagal inhibition that increases HR (Fig. 2A) while decreasing its variability, great RSAS decrease (Fig. 2E) with progressive uncoupling of the respiratory modulation (Fig. 2F) and large progressive BRS reduction (Fig. 2C), also with gradual input-output uncoupling (Fig. 2D); 2) gradual sympathetic activation associated to DP increment, reinforced by the metaboreflex (MBR) activation, whose onset is indicated by the threshold effect presented in the last third of LIFIE (Fig. 2B); and 3) respiratory activation leading to increased ventilation, triggered in the last third of LIFIE (Fig. 2G), associated to MBR activation and probably to anaerobic acidosis. Almost at the end of LIFIE, cBRS and cRSAS show a threshold effect with tendency to recover (Fig. 2D and F) that can be attributed to the sympathetic increase by the MBR activation and a mechanical modulatory effect of Res, respectively. Thus, the progressive resetting and uncoupling of RSA and BR mechanisms contribute the progressive release of sympathetic activity and Res from their restraint.

Despite that $\mathrm{HG}$ and $\mathrm{LE}$ are performed at the same rate of MF increase and attain similar maximal MF, the latter provokes greater BRS, HR and DP effects and stronger correlations with \% MF than $\mathrm{HG}$, effects attributed to the greater muscular mass involved in LE [4] but that can also be explained by the different $\mathrm{CC}$ drive of ACR function, depending on whether it activates the muscles of the upper or lower limbs.

In conclusion, RSAS, an index of RSA that considers the respiratory influence, can be computed by alpha index, analogously to BRS, and their coherences may be used as measures of the instantaneous degree of their input-output coupling. The inverse relationships of $\% \mathrm{MF}$ with RSAS, cRSAS, BRS and cBRS, show moderate to strong correlations, with greater slopes in LE for BRS, due to the different $\mathrm{CC}$ drive of upper and lower limbs, which, by gradually suppressing sensitivities and coherences, provokes progressive vagal withdrawal and loss of respiratory-cardiac modulation that causes greater regularity in HR increase and allow the release of sympathetic and respiratory drives as necessary conditions for the parallel rise of DP, HR and ventilation.

\section{References}

[1] Figueroa A, Hooshmand S, Figueroa M, Bada A. Cardiovagal baroreflex and aortic hemodynamic responses to isometric exercise and post-exercise muscle ischemia in resistance trained men. Scand J Med Sci Sports 2010;20:305-9.

[2] Florian J, Simmons E, Chon K, Faes L, Shykoff B. Cardiovascular and autonomic responses to physiological stressors before and after six hours of water immersion. J Appl Physiol 2013;115:1275-89.

[3] Iellamo F, Massaro M, Raimondi G, Peruzzi G, Legramante J. Role of muscular factors in cardiorespiratory responses to static exercise: contribution of reflex mechanisms. J Appl Physiol 1999;86:174-80.

[4] Saul J, Berger R, Chen M, Cohen R. Transfer function analysis of autonomic regulation. II. Respiratory sinus arrhythmia. Am J Physiol 1989;256:H153-61.

[5] Pagani M, Somers V, Furlan R, Dell'Orto S, et al. Changes in autonomic regulation induced by physical training in mild hypertension. Hypertension 1988;12:600-10.

[6] Heffernan K, Baynard T, Goulopoulou S, Giannopoulou I, et al. Baroreflex sensitivity during static exercise in individuals with Down Syndrome. Med Sci Sports Exerc 2005;37:2026-31.

[7] Stewart J, Montgomery L, Glover J, Medow M. Changes in regional blood volume and blood flow during static handgrip. Am J Physiol Heart Circ Physiol 2007;292:215-23.

[8] Mancia G, Parati G, Castiglioni P, di Rienzo M. Effect of sinoaortic denervation on frequency-domain estimates of baroreflex sensitivity in conscious cats. Am J Physiol 1999;276:H1987-93.

Address for correspondence.

Alejandra Guillén-Mandujano

Depto. Ciencias de la Salud, T-172. UAM-I, CDMX, México. ale.guillen.mandujano@gmail.com 